\title{
Design and Implementation of Vehicle Electrical Terminals Based on Android
}

\author{
Yang $\mathrm{Xu}^{1,}$, Heping Gong ${ }^{2, \mathrm{~b}}$ \\ ${ }^{1}$ School of Automation, Chongqing University of Posts and Telecommunications, Chongqing \\ 400065, China; \\ ${ }^{2}$ School of Automation, Chongqing University of Posts and Telecommunications, Chongqing \\ 400065, China; \\ a342870@qq.com, b1510078914@qq.com
}

Keywords: Android, Electric vehicle, Terminal, Monitoring system.

\begin{abstract}
In this paper, the terminal for the electric vehicles based on Android system is designed. In the system, PX2 is used as the core processor, together with STM32 and other peripheral control chips. A lot of third-party entertainment applications are integrated into the terminal. At the same time, in order to achieve effective monitoring of the running state on the electric vehicle, this paper mainly focuses on the design and implementation of the electrical vehicle monitoring system. The results of the testing prove that the designing targets are achieved, and the system not only meet the entertainment needs, but also complete the monitoring function of the electric vehicle in the background.
\end{abstract}

\section{Introduction}

In recent years, with the development of electric vehicles, the development and operation of the vehicle electrical terminal's ancillary products undoubtedly play a positive role in the improvement of the industry. A high quality entertainment and reliable security environment for people in the limited space has become a focus recently. The running state of the electric vehicle is influenced by many kinds of factors in the running process of the vehicle, such as voltage, electric current. Researchers at home and abroad to spend a lot of time researching the design and implementation of vehicle electrical terminals and have achieved certain results[1][2]. Due to the Android system has the advantages of open source code, complete multimedia, network and graphics, in line with the requirements of the vehicle network to the end terminal system[3]. Therefore, according to the characteristics of electric vehicle, this paper introduces the hardware and software of the terminal for the electric vehicles which based on Android system, the design can not only meet the entertainment experience of driving but also upload CAN and GPS data to the server efficiently by using $3 \mathrm{G}$ mobile network to ensure the user's safe driving.

\section{The overall function design of the system}

By taking into account the needs of actual operation, the terminal system are integrated with the Map Navigation, Car radio, Bluetooth phone, Audio and Video, External input, Screen sharing, in addition, the design of the monitoring system of electric vehicle is designed specially. The overall design structure of the terminal is shown in Fig.1. 




Fig.1 The overall design structure of the terminal

\section{System hardware design}

By vehicle electrical terminal function the characteristics of the ARM host processor hardware resources in-depth analysis, based on a simplified system design, improving system integration and reliability, to facilitate system software development principles, to determine the hardware selection of the terminal and program determined. The vehicle terminal hardware mainly include the following modules: ARM host processor, 3G module, GPS module, the CAN core data acquisition board, Bluetooth module, WIFI module, Memory module and Power supply module, etc. Hardware block-diagram of vehicle terminal is shown in Fig.2.



Fig.2 Hardware block-diagram of vehicle terminal

PX2 produced by Rockchip company is able to meet the requirements of the hardware environment for Android system, the interfaces between the microcontroller and the external modules are provided. The Huawei MU509 3G module is connected to PX2 core board through the USB, the module is mainly responsible for providing efficient network on the vehicle. The system connects NEO-6 module with the PX2 chip through the UART interface and achieve the goal of obtaining GPS information according to NMEA protocol. The CAN data acquisition core board which adopt STM32f103RB8 as the core chip upload the electric vehicle CAN data to the PX2 through the MCU serial port after receiving and converting them. The Bluetooth module produced by Shenzhen GuKai Company is connected with the PX2 core board through the serial port, it can achieve Bluetooth phone function by sending AT commands to the module from the Android display terminal. The WIFI module named USB RTL8188EUS W12 through the USB interface with the PX2 core board carry out a physical connection, and provide WIFI connection support. The range of voltage 9 16V is supplied by the power supply and the overvoltage protection circuit is designed to ensure the safety of the terminal when the voltage exceeds $16 \mathrm{~V}$. 


\section{Software design of the system}

\subsection{Construction of the Android software development platform.}

The system relates to specific hardware access, it is necessary to configure the development environment on Linux Unbuntu 14.04 operation system. First of all, the Java Development Kits (JDK) is installed to ensure that the Java program can be compiled, and the make compiler version is 3.8. The Android SDK platform is installed, which provides tools for developing Android application, then the open source code for the Android 4.4 system is obtained. Once the source files have been successfully configured, using the make command to compile the Android source code. Then, extensible applications software to be programmed using the platform and be localized in the system source code path/packages/apps to compile. The APK file would be generated under the /out/target/product/rkpx2/system/app path, then push APK file to the terminal for debugging and running through the Android debug bridge ADB tool, which is provided in the Android SDK.

\subsection{Android hardware driver.}

In the design of the terminal system, Unbuntu 14.04 LTS operating system was used in host computer. Due to the dependency of embedded software on the target hardware platform, it can not be compiled and operated under the host environment, so the cross compiler environment was established in the platform in order to compile a program to run in another architecture. The bootloader of Android can complete the initialization of the main components of the system board, and provide an appropriate hardware and software environment for the normal startup system.

The system mainly includes the transplantation of the driver and the file system. The application can call the corresponding Application Programming Interface(API) after the driver is transplanted. For the 3G module driver, firstly, adding 3G module driver and PPP network protocol to the Linux kernel. Secondly, ppp-2.4.4 source code will be cross-compiled. For the GPS transplantation, the first task is to realize the serial port driver based on TTY in driver layer. Ublox company provides us the HAL layer driver code, the code eventually generate .so file, the file not only provides analytic GPS data to the GPS framework layer but also set up the receiver GPS to get raw GPS data through the interface (which can be UART or I2C).

\subsection{Terminal software integration.}

A lot of practical software was integrated into the Android platform in order to facilitate a more user friendly experience of driving. The system uses sophisticated A-map to display map information, saving development costs and improve efficiency. In addition to this, other entertainment application were' developed according to hardware and software resources that existed in the system, such as Radio, Bluetooth Phone, AV, Screen sharing, etc.

\subsection{Terminal Monitoring software design}

Platform exchange communication protocol. Communication protocol between vehicle terminal and background monitoring server is defined in the vehicle monitoring system. The protocol uses big endian mode of network byte order to pass word and double word, packaged data would be sent to the server through the $3 \mathrm{G}$ network in $\mathrm{C} / \mathrm{S}$ mode. The frame format of the transmitted data packet is shown in Table 1.

Table 1 Frame format of the transmitted data packet

\begin{tabular}{|c|c|c|c|c|c|c|}
\hline $\begin{array}{c}\text { Start } \\
\text { symbol }\end{array}$ & $\begin{array}{c}\text { Command } \\
\text { unit }\end{array}$ & $\begin{array}{c}\text { Identification } \\
\text { code }\end{array}$ & $\begin{array}{c}\text { Data } \\
\text { encryption } \\
\text { mode }\end{array}$ & Data unit length & Data unit & $\begin{array}{c}\text { Checkin } \\
\text { g code }\end{array}$ \\
\hline
\end{tabular}

The message format begins with start symbol, the subsequent including command unit, identification code, data encryption mode, data unit length, data unit and check code. The start symbol was fixed to the ASCII character '\#\#' or "0x23, 0x23".Command unit from the server was sent to the car terminal set in order to modify the parameter information of vehicle terminal. The vehicle identification code is the only identification, consisting of 17 bit code. There are four ways of data encryption: ' $0 \mathrm{x} 01$ ' indicates that the data was not encrypted, ' $\mathrm{x} 02$ ' indicates that the data was encrypted by RSA algorithm, ' $0 \mathrm{x} 03$ ' indicates that the data was encrypted by the AES128 bit 
algorithm, '0xFE' represents an exception, and "0xFF" is invalid. The data unit includes data acquisition time, information type mark and information body, wherein the information body includes the electric parameters of the power battery, the temperature data of the power battery pack, the fuel cell data and the vehicle data and so on. BCC method was applied in checking code, calibration range start from the first byte of command unit, with one byte XOR, until the previous check code. Checking code takes up one byte.

The design of monitoring application program for electric vehicles. The monitoring system that based on Android take into account the needs to run automatically when the electric mobile starts firing. This application should boot up as Android system's service. The CAN information from the data acquisition module includes the battery information, energy feedback frequency, motor speed and alarm, monomer voltage information, monomer temperature, time and charge for weeks, charger output information and so on, they will be sent to PX2 core broad through micro controller serial port and can be registered as a broadcast on Android platform. CAN information would be obtained through broadcast receiver in the application. GPS information acquisition mainly use the geographic location services in the framework of the Android system and network to obtain the precise positioning of the electric vehicle. The server analysis the data after receiving the information sent by the terminal server, and display the running state of the electric vehicle according to the actual needs in the background. Software flow chart of electric vehicle monitoring system is shown in Figure 3.

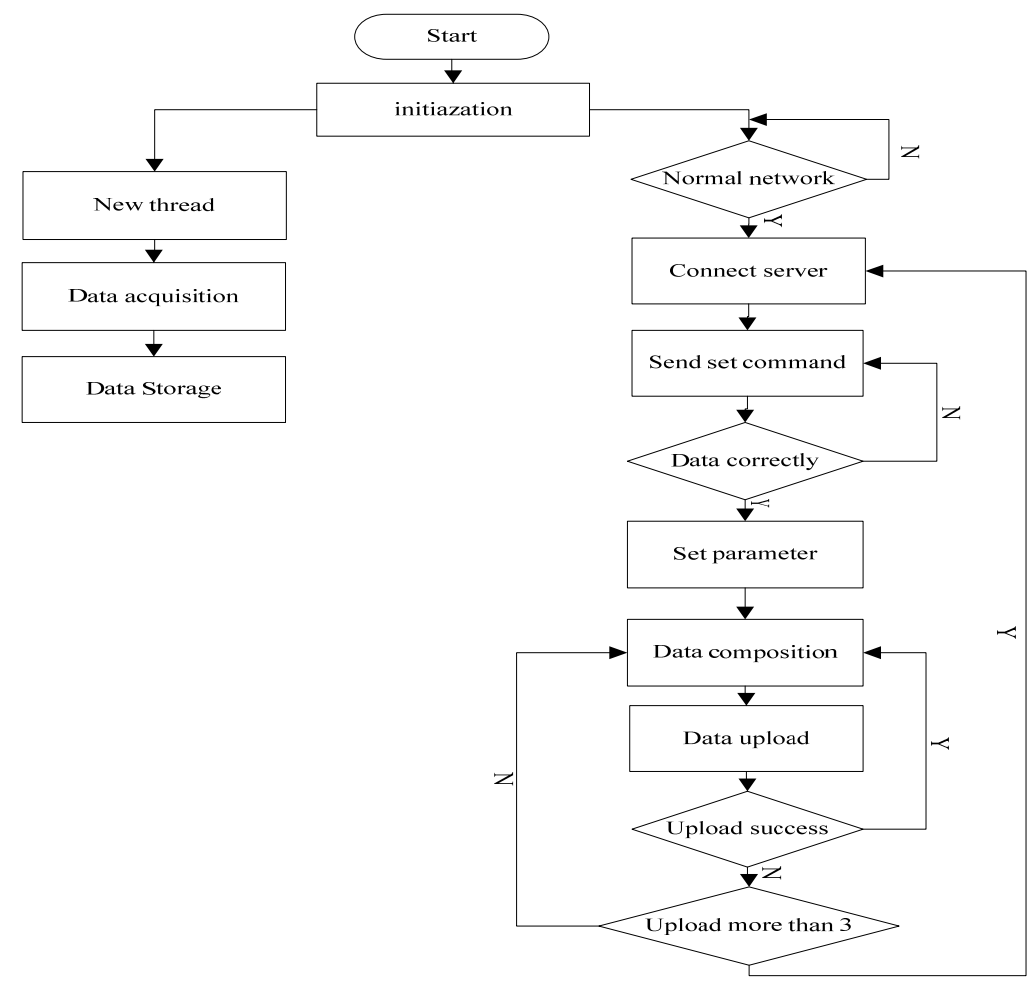

Fig.3 Software flow chart of electric vehicle monitoring system

First of all, the initialization of the monitor is responsible for starting monitor service. Secondly, building a new thread to collect CAN\&GPS data every second and checking whether the network is normal, then, establishing connection with the server through the network IP address and port number if the network is available. Thirdly, obtaining sample frequency and system clock by sending data packet of terminal's set to the server, next, upload GPS \&CAN data to the server after packaging them according to the data communication protocol. Once the data have been uploaded successfully, continue the next transfer in a circular fashion, otherwise, check whether it is normal of the network if the data transfer more than three times. 


\section{Test verification}

The vehicle electrical display terminal is installed on the electric vehicle car, and around the school in a laboratory building outdoor experiments. The test shows that the terminal performance well on the electric vehicle car, it can meet the entertainment needs and complete the data transmission effectively. The overall effect diagram of vehicle electrical display terminal and the parsed data on the server side are shown in Fig.4 and Fig.5.



Fig.4 The overall effect diagram of vehicle electrical display terminal

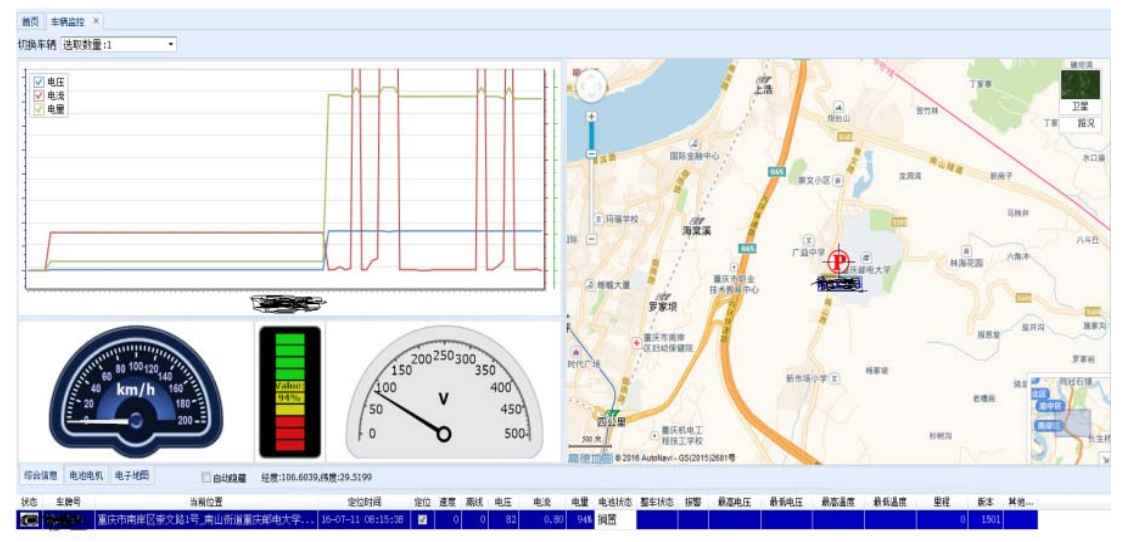

Fig.5 Parsed data on the server side

\section{Summary}

This paper introduce the design of the hardware and software of the electric vehicle display terminal. Electric vehicle monitoring based on Android system are especially introduced. The actual test prove that the terminal has achieved the needs of entertainment and safety monitoring on electric vehicles, and gives the actual operating results. It will be widely applied.

\section{Acknowledgments}

It is a project supported by Achievement Transfer Program of Institutions of Higher Education in Chongqing (KJZH14207) and Student Research Training Program(A2015-78). 


\section{References}

[1] J.H. Zheng,T. Wei,Research and Design of Vehicle Mobile Terminal Based on Android, J. Applied Mechanics \& Materials.441(2013)924-927.

[2] F. Simonot-Lion, The design of safe automotive electronic systems: some problems, solutions and open issues,J. IEEE Symposium on Industrial Embedded Systems Ies. 2006.

[3] X.C. Yang,J.T. Hu,Y.Q. Dong,Display Terminal of Electric Vehicle In-Vehicle Information System Based on Android ,J. Journal of Nanchang University.2011.

[4] M.J. Kim,M. Ali,T. Menard,Determining time to traverse road sections based on mapping discrete GPS vehicle data to continuous flows, Proc. of IEEE Intelligent Vehicles Symbosium (IV).2010,pp. 615-620.

[5] L. Li,X. Lu,J. He,Z. Sun,G. Yang,Design of New-generation Electric Vehicle Terminal,J.Energy \& Power Engineering.5(2013)1362-1366.

[6] Information on http://www.spsp.gov.cn 